\title{
Comparative Study between Efficacy of Serratus Anterior Muscle Block as A Regional Analgesia Technique and I.V Morphine Infusion in Patient with Fracture Ribs \\ Wesam Abd El-Galil Abu-Elwafa, Islam Ahmed Ragab*, Abdelrahman Hassan Abdelrahman, Wael Alham Mahmoud
}

Department of Anaesthesia, ICU \& Pain Management, Sohag Faculty of Medicine, Sohag University *Corresponding author: Wesam Abd El-Galil Abu-Elwafa, Mobile: (+20) 01002005802,

E-Mail: abdelgalilwesam@gmail.com

\begin{abstract}
Background: Rib fractures are very common and are detected in at least $10 \%$ of all injured patients, the majority of which are because of blunt thoracic trauma with road traffic collisions being the main cause. Pain management of the acutely injured patient with rib fractures can be difficult for even the most experienced emergency physician.

Objective: To evaluate the safety and efficacy of serratus anterior muscle plane block as a regional analgesic technique performed in patient with fracture ribs.

Patients and Methods: Randomized, controlled study was carried out in Department of Anesthesia and Intensive Care Unit, Sohag University Hospital. Forty patients with lateral multiple rib fractures were included in this study. All patients were suffering from excruciating pain and were not responding to conventional analgesics. The patients were randomly assigned into two groups, 20 patients each. Group A, for whom serratus anterior plane block (SAP) was done and Group B, for IV morphine infusion.

Results: In Group A, the mean pain score decreased from $9.1 \pm 0.7$ to $0.6 \pm 0.7$ after intervention. In group B, the mean pain score improved from $9.3 \pm 0.8$ to $1.15 \pm 0.8$ after IV morphine infusion. Pain scores gradually decreased. There were no significant difference between the two groups when the other trauma surveys were compared.

Conclusion: Ultrasound guided Serratus Anterior Plane block is an effective technique for providing analgesia in patients with anterior and lateral rib fractures with fewer risks of complications.
\end{abstract}

Keywords: Regional analgesia, Serratus anterior muscle block, I.V Morphine infusion.

\section{INTRODUCTION}

Rib fractures are very common and are detected in at least $10 \%$ of all injured patients, the majority of which are because of blunt thoracic trauma $(75 \%)$ with road traffic collisions being the main cause. The remaining $(25 \%)$ are due to penetrating injuries. Rib fractures are associated with significant morbidity, with patients frequently requiring admission to the intensive care unit (ICU), and mortality rates as high as $(33 \%)^{(\mathbf{1})}$.

Morphine is a pain medication of the opiate family, which is found naturally in a number of plants and animals. It acts directly on the central nervous system (CNS) to decrease the feeling of pain. It can be taken for both acute pain and chronic pain. It is frequently used for pain from myocardial infarction and during labor. It can be given by mouth, by injection into a muscle, by injection under the skin, intravenously, injection into the space around the spinal cord, or rectally ${ }^{(2)}$. Esmailian et al. ${ }^{(3)}$ showed that morphine has a therapeutic value in relieving the pain of rib fracture. The success rate after 30 minutes drug administration was $58.6 \%$.

Ultrasound-guided serratus anterior plane (SAP) block is a relatively new technique that provides analgesia for thoracic wall surgeries by blocking the lateral branches of the intercostal nerves (T2-L2). The procedure has been largely discussed for managing pain after breast surgery, and new interest has arisen for the treatment of thoracic trauma, specifically rib fractures. Inadequate pain management of rib fractures causes significant morbidity and mortality of rib fractures, particularly the development of pneumonia ${ }^{(4)}$. Jadon and Jain ${ }^{(5)}$ concluded that serratus anterior plane block could provide effective analgesia in patients with multiple rib fractures. However, the results need to be further substantiated in randomised controlled studies with larger number of patients comparing the efficacy of this block with other regional anaesthetic techniques and evaluate the efficacy of Serratus Plane Block for thoracic wall blockade if fractures are occurring in posterior $1 / 3$ part.

\section{AIM OF THE WORK}

Evaluate the safety and efficacy of serratus anterior muscle block as a regional analgesia technique performed in patient with fracture ribs by the use of ultrasound to improve the success rate and accuracy of the Serratus anterior block \& to prevent potential complications.

\section{PATIENTS AND METHODS}

This was an interventional, randomized and controlled study that was carried out in Department of Anesthesia \& Intensive Care Unit, Sohag University Hospital.

Ethical and patients' approval: After an approval of the Ethics Committee of Sohag University and 
according to the guidelines noted in the World Health Organization chronicles in 1976, written and oral consents were obtained from the patients after explaining the benefits and the hazards of the maneuver.

Forty patients with lateral and/or anterior multiple rib fractures (3 to 6 in numbers) were included in this study. All patients were suffering from excruciating pain and were not responding to conventional analgesics. The patients were randomly assigned into two groups, 20 patients each:

Group A: Patients whom serratus anterior plane block was done.

Group B: Patients that received I.V Morphine (0.1 $\mathrm{mg} / \mathrm{kg}$ as a loading dose, $10-20 \mathrm{ug} / \mathrm{kg} / \mathrm{hr}$ as an infusion). -The use of the Visual Analogue Scale (VAS) was explained to all patients (in which 0 represents no pain and 10 the worst imaginable pain).

\section{Inclusion criteria:}

1. Lateral, anterior rib fracture.

2. Hemodynamically stable.

3. Fully conscious.

4. Minimal to mild hemothorax or pneumothorax.

5. Older than 15 years.

6. Both sexes.

7. ASA (American Society of Anaesthesiologist) physical status classification system I to III.

8. Possibility of anatomical structures ultrasound identification in a satisfactory way.

\section{Exclusion criteria:}

1. Heamodynamic instability.

2. ASA (American Society of Anesthesiologist) physical status classification system IV and V.

3. Morbid obesity (body mass index $>40$ ).

4. Impossibility of anatomical structures identification by ultrasound in a satisfactory way (there can be no distinction in the interfascial plane between serratus and latissimus dorsi muscle).

5. Infection at the puncture site.

6. Haemostasis disorders.

7. Allergy to any of the drugs used in the study.

8. Inability to communicate with the investigator.

9. Underlying lung injury.

\section{Procedure:}

Standard non-invasive monitoring should be applied, pulse oximetry, noninvasive blood pressure, 5 lead electrocardiogram and an i.v. cannula inserted. Aseptic precautions should be maintained throughout the procedure.

In the study group patients, an ultrasound guided serratus anterior plane block was performed with the patient in the supine position and the arm abducted. Using a high-frequency linear ultrasound probe set between 6 and $13 \mathrm{MHz}$, place the probe in the sagittal plane and identify the fifth rib in the mid-axillary line.
Latissimus dorsi and serratus anterior muscles are now easily identifiable overlying the fifth rib.

A 22-gauge, 90-mm spinal needle (TeaChang Industrial Co., Ltd., Korea) was introduced targeting the plane superficial to the serratus anterior muscle aspiration, and then $15 \mathrm{~mL}$ of $0.25 \%$ bupivacaine plus $15 \mathrm{ml}$ of $1 \%$ lignocaine were injected under continuous ultrasound guidance. Then, the needle was removed. During the injection, the patients were closely monitored for any signs of local anaesthetic toxicity (tachycardia, arrhythmias, altered consciousness, and seizures). Aggressive respiratory physiotherapy and early mobilization were encouraged in the patients once pain was (4) or less on deep breathing.

In the control group patients, IV access line for i.v. morphine $[(0.1 \mathrm{mg} / \mathrm{kg}$ as a loading dose, (10-20 $\mathrm{ug} / \mathrm{kg} / \mathrm{hr}$ ) as an infusion] keeps the VAS scores less than (3).

Primary outcome measures: Quality of analgesia measured by visual analogue scale to rate pain severity scored from 0 to 10 ; while 0 stands for no pain at all and 10 is the worst intolerable pain.

\section{Secondary outcome measures:}

- Opioid-related complications (nausea, vomiting, apnea, urinary retention and/or ileus).

- Signs of local anesthesia toxicity e.g. circumoral numbness, tongue paraesthesia, etc.

- Possible complications related to the maneuver like (infection, hematoma, nerve injury or pleural injury).

- ICU stay time.

\section{Statistical analysis}

Data were verified, coded by the researcher and analyzed using IBM-SPSS Statistics for windows, version 23.0 (Copyright IBM Corp., Armonk, N.Y., USA. 2015). Descriptive statistics: Mean, standard deviation, median, range and percentage were calculated.

Test of significances: Chi-square test was used to compare the difference in distribution of frequencies among different groups. For continuous variables, independent t-test analysis was carried out to compare the means of dichotomous data. There was no specific calculation of the sample size. A significant p-value was considered when it is equal or less than 0.05 .

\section{RESULTS}

Regarding pulse, there were statistically high significant differences between the two groups regarding before injection $(\mathrm{P}$ value $<0.001)$, after injection $(\mathrm{P}$ value $<0.001)$, after 3 hours $(\mathrm{P}$ value < $0.001)$, after 6 hours ( $\mathrm{P}$ value $<0.001)$, after 12 hours ( $\mathrm{P}$ value $<0.001)$ and after 18 hours ( $\mathrm{P}$ value $<0.001)$, and a significant difference regarding after 24 hours ( $\mathrm{P}$ value 0.003 ) as shown in table (1). 
Table (1): Changes in pulse before injection, after injection, $3 \mathrm{hr}, 6 \mathrm{hr}, 12 \mathrm{hr}, 18 \mathrm{hr}$ and $24 \mathrm{hr}$ after injection as compared between the two groups

\begin{tabular}{lcccc}
\hline \multicolumn{1}{c}{ Pulse } & Group A $(\mathbf{n = 2 0})$ & Group B $(\mathbf{n = 2 0})$ & $\mathbf{T}$ & $\mathbf{P}$ \\
\hline Before injection & $112.8 \pm 8.4$ & $121.7 \pm 5.7$ & 3.9 & $<0.001$ (HS) \\
After injection & $79.9 \pm 4.2$ & $97.2 \pm 6.1$ & 10.3 & $<0.001$ (HS) \\
3 hours & $78.4 \pm 4.6$ & $92.1 \pm 5.3$ & 8.6 & $<0.001$ (HS) \\
6 hours & $77.7 \pm 4.9$ & $89.8 \pm 4.6$ & 8 & $<0.001$ (HS) \\
12 hours & $77.9 \pm 4.6$ & $88.5 \pm 3.7$ & 8 & $<0.001$ (HS) \\
18 hours & $79.2 \pm 3.7$ & $86.9 \pm 3.7$ & 6.5 & $<0.001$ (HS) \\
24 hours & $82.3 \pm 2.9$ & $85.8 \pm 4.1$ & 3.1 & 0.003 (S) \\
\hline
\end{tabular}

Regarding systolic blood pressure, there were non-significant difference between the two studied groups throughout the study period (Table 2).

Table (2): Changes in systolic blood pressure (SBP) before injection, after injection, $3 \mathrm{hr}, 6 \mathrm{hr}, 12 \mathrm{hr}, 18 \mathrm{hr}$ and 24hr after injection as compared between the two groups

\begin{tabular}{lcccc}
\hline \multicolumn{1}{c}{ SBP } & Group A $(\mathbf{n}=\mathbf{2 0})$ & Group B $(\mathbf{n}=\mathbf{2 0})$ & $\mathbf{T}$ & $\mathbf{P}$ \\
\hline Before injection & $133.5 \pm 7.9$ & $137.2 \pm 8.9$ & 1.39 & $0.16(\mathrm{NS})$ \\
After injection & $123.5 \pm 7.1$ & $127.2 \pm 8.6$ & 1.49 & $0.14(\mathrm{NS})$ \\
3 hours & $121.5 \pm 6.1$ & $124 \pm 7.4$ & 1.16 & $0.24(\mathrm{NS})$ \\
6 hours & $120.7 \pm 5.4$ & $124 \pm 6.4$ & 1.72 & $0.09(\mathrm{NS})$ \\
$\mathbf{1 2}$ hours & $120.7 \pm 4.9$ & $123.5 \pm 4.9$ & 1.76 & $0.08(\mathrm{NS})$ \\
$\mathbf{1 8}$ hours & $120.7 \pm 5.4$ & $122.5 \pm 5.5$ & 1.01 & $0.31(\mathrm{NS})$ \\
$\mathbf{2 4}$ hours & $120.7 \pm 3.7$ & $121 \pm 5$ & 0.17 & $0.85(\mathrm{NS})$ \\
\hline
\end{tabular}

Regarding diastolic blood pressure, there were non-significant difference between the two studied groups throughout the study period (Table 3 ).

Table (3): Changes in diastolic blood pressure (DBP) before injection, after injection, $3 \mathrm{hr}, 6 \mathrm{hr}, 12 \mathrm{hr}, 18 \mathrm{hr}$ and 24 hr after injection ass compared between two groups

\begin{tabular}{lcccc}
\hline \multicolumn{1}{c}{ DBP } & Group A $(\mathbf{n}=\mathbf{2 0})$ & Group B $(\mathbf{n}=\mathbf{2 0})$ & $\mathbf{T}$ & $\mathbf{P}$ \\
\hline Before injection & $89 \pm 5.9$ & $90 \pm 5.8$ & 0.53 & $0.59(\mathrm{NS})$ \\
After injection & $83 \pm 4.4$ & $82 \pm 4.9$ & 0.67 & $0.5(\mathrm{NS})$ \\
3 hours & $81 \pm 2.6$ & $81.2 \pm 2.75$ & 0.29 & $0.76(\mathrm{NS})$ \\
6 hours & $79 \pm 2.6$ & $80.2 \pm 3$ & 1.39 & $0.17(\mathrm{NS})$ \\
$\mathbf{1 2}$ hours & $79 \pm 2.6$ & $80.5 \pm 3.2$ & 1.62 & $0.11(\mathrm{NS})$ \\
$\mathbf{1 8}$ hours & $79 \pm 2.6$ & $79.7 \pm 2.6$ & 0.91 & $0.36(\mathrm{NS})$ \\
$\mathbf{2 4}$ hours & $78.5 \pm 2.85$ & $79.7 \pm 3$ & 1.34 & $0.18(\mathrm{NS})$ \\
\hline
\end{tabular}

There was no significant difference between the two groups regarding analgesia demand (Table 4). However, there was only 1 patient in group A needed combination analgesia ketolac $16 \mathrm{mg}$ in 2 doses and paracetamol 1000 mg one dose.

Table (4): Analgesia demand if the patient need another type of analgesia to control pain like ketorolac or paracetamol or both of them

\begin{tabular}{lcccccc}
\hline & \multicolumn{2}{c}{ Group A } & \multicolumn{2}{c}{ Group B } & \multirow{2}{*}{$\mathbf{X}^{\mathbf{2}}$} & \multirow{2}{*}{ P } \\
\cline { 2 - 5 } & No & $\mathbf{\%}$ & No & \% & & \\
\hline Paracetamol & 1 & 5 & 0 & 0 & 1.44 & $0.2(\mathrm{NS})$ \\
Ketorolac & 1 & 5 & 0 & 0 & 1.44 & $0.2(\mathrm{NS})$ \\
Combination & 1 & 5 & 0 & 0 & 1.44 & $0.2(\mathrm{NS})$ \\
\hline
\end{tabular}




\section{DISCUSSION}

Pain caused by fracture of one or two ribs is usually controlled efficiently by oral or systemic analgesic drugs; However pain accompanying multiple rib fractures (three ribs or more) was mostly severe, difficult to be controlled and may limit the ability to breathe, cough and even movement ${ }^{(6)}$. Rib fractures cause severe pain and contribute significantly to the morbidity and mortality presented in polytrauma patients particularly in those with poor respiratory reserve. Inability to cough and breathe deeply due to severe pain leads to sputum retention, atelectasis and so reduction in the functional residual capacity. These factors in turn lead to decreased lung compliance, ventilation perfusion mismatch, hypoxemia and respiratory distress. Therefore effective pain relief remains the cornerstone of management in these cases to prevent serious respiratory complications. Opioidbased analgesic techniques are effective but have inherent limitations of respiratory reserve together with other complications, which are dose dependent. therefore, regional analgesic techniques are a preferred choice over opioids for pain relief in rib fractures. Thoracic epidural analgesia and thoracic paravertebral block are effective techniques in management of these cases. However, these techniques are more invasive and associated with various adverse effects. Other regional techniques like intrapleural block and intercostal nerve blocks have variable success and high potential for local anesthetic toxicity ${ }^{(7)}$.

Ultrasound guided serratus anterior plane block is a relatively novel technique that is less invasive, easier to be performed and has a better safety profile. This technique was initially described for breast surgeries by Blanco et al. ${ }^{(8)}$ where it can achieve paresthesia of the complete hemithorax.

In our study, we aimed to evaluate the safety and efficacy of ultrasound guided serratus anterior muscle block as a regional analgesic technique performed in patients with multiple fracture ribs. The use of ultrasound was introduced to improve the success rate and accuracy of the serratus anterior block and to prevent potential complications.

Regarding demographic data, our study showed that there was no significant difference between the two groups concerning age, gender and weight of the patients.

As regards pain scores in our study, the mean pain score in group A before block was $9.1 \pm 0.7$ and after block was $0.6 \pm 0.7$. In Group B, the mean pain score before i.v. morphine infusion was $9.3 \pm 0.8$, and after i.v. morphine infusion was $1.15 \pm 0.8$, there were significant differences between the two groups. In agreement with our study Peter and Paul (9), found that after injection of $40 \mathrm{ml}$ ropivacaine $0.25 \%$ in a patient with multiple fracture ribs; immediate symptomatic relief of pain and NRS (numerical rating scale) decreased from 7 to 0 . Also, Matteo et al. ${ }^{(10)}$, found that after administration of $15 \mathrm{~mL}$ of ropivacaine $0.125 \%+$ $15 \mathrm{~mL}$ of lignocaine $1 \%$ in a patient with multiple rib fractures, the NRS pain score diminished from 10 to 2 . In contrast with our study, Jadon and Jain ${ }^{(5)}$ reported that in patients with unilateral multiple rib fractures, serratus anterior plane block under ultrasound guidance with a bolus dose of $20 \mathrm{ml} 0.5 \%$ ropivacaine between the serratus anterior and external intercostal muscle they found that the median pain score on deep breathing before block was $8.5 \pm 1.211$ while median pain score on deep breathing after block was $3.0 \pm 0.894$; which might be due to injection of lower volume than our study $(20 \mathrm{ml})$ and the use of $0.5 \%$ ropivacaine alone while we used a mixture of bupivacaine and lignocaine and the technique of the block was below the serratus muscle (deep serratus block), which had lower analgesic effect than superficial serratus block in our study.

Regarding onset of analgesia, in our study we found that there was a statistically high significant difference between the two groups with more rapid onset in group A $(1.3 \pm 0.47 \mathrm{~min})$ than in group B $(5.4 \pm 2.3$ min) as after SAPB in group A there was nearly immediate symptomatic relief of pain. These results are in agreement with, Peter and Paul ${ }^{(9)}$ who reported that after injection of $40 \mathrm{~mL}$ ropivacaine $0.25 \%$ in a patient with multiple fracture ribs they found immediate symptomatic relief of pain and NRS (numerical rating scale) score decreased from 7 to 0 . In contrast to our study in a case report, Kunhabdulla et al. ${ }^{(11)}$ reported that after injection of $20 \mathrm{~mL}$ of $0.125 \%$ bupivacaine through SABP for patient with fracture ribs they found that the patient showed a significant decrease in pain 15 minutes after the procedure. This might be explained by the lower volume used so it took a longer time to start action, lower concentration of local anesthetic $(0.125 \%)$ and use of bupivacaine as a sole agent with its relatively slow onset of action. Also, In a case report by Rose et al. ${ }^{(\mathbf{1 2})}$ after injection of $30 \mathrm{~mL}$ of ropivacaine $0.5 \%$ with epinephrine $1: 400,000$ below the serratus muscle (in the fascial plane between the serratus muscle and rib periosteum or external intercostal muscle), they noticed that the patient was able to inspire deeply and cough efficiently with minimal pain within $15 \mathrm{~min}$ of the procedure. this might be due to the technique used (injection was below serratus muscle) and the use of ropivacaine $0.5 \%$ concentration as a sole agent. Moreover, in a case report by Okmen et al. ${ }^{(13)}$ on SPB for post-thoracotomy pain, they reported that pain relief after $10 \mathrm{~min}$ from injecting $6 \mathrm{ml}$ of $1 \%$ lidocaine followed by continuous infusion of $0.1 \%$ bupivacaine at $7 \mathrm{ml} / \mathrm{h}$. The delay of onset in this case was surly due to starting by low volume and lower concentration of local anesthetic ( $6 \mathrm{ml}$ of lidocaine $1 \%)$.

As regards duration of analgesia in our study, there were no significant differences between both groups. In group A, the mean was $49.6 \pm 7.7 \mathrm{hr}$, which 
was close to group B mean $(50.4 \pm 4.9 \mathrm{hr})$. In agreement with our study, Fady et al. ${ }^{(14)}$ compared ultrasound guided serratus anterior plane block versus thoracic paravertebral block (PVB) for perioperative analgesia in thoracotomy after injection of $30 \mathrm{ml}$ of $0.5 \%$ bupivacaine in SABP, they noticed that analgesia was adequate in SAPB groups up to $24 \mathrm{~h}$ after surgery. Also, Samar et al. ${ }^{(15)}$ found that the duration of analgesia was $20 \pm 3 \mathrm{~h}$ ) for patients who received serratus intercostal plane block with $0.4 \mathrm{ml} / \mathrm{kg}$ bupivacaine $0.25 \%$ plus adrenaline $5 \mu \mathrm{g} / \mathrm{ml}$ as a single injection at the end of mastectomy operation. In contrast with our study, in a case report by Ashok (16) after injection of $20 \mathrm{ml}$ of $0.25 \%$ bupivacaine with adrenaline $(5 \mu \mathrm{g} / \mathrm{ml})$ given as a bolus below the serratus muscle for patients with multiple fracture ribs. They found that the pain relief lasted until $6 \mathrm{hrs}$, which might be explained by that the injection was below serratus and the volume of LA (20 $\mathrm{ml}$ of $0.25 \%$ bupivacaine), which is likely an important determinant of the extent and duration of analgesia for (SAPB). Since, SAPB is a fascial block, a larger volume is expected to promote more LA spread and longer analgesic duration.

Also, Okmen et al. ${ }^{(13)}$ in their case study reported that the duration of analgesia obtained by the (SPB) lasted for $7 \mathrm{~h}$ after injecting $6 \mathrm{ml}$ of $1 \%$ lidocaine this surely explained by volume, concentration and type of L.A which was injected (6 $\mathrm{ml}$ of $1 \%$ of short acting lidocaine). Also, Gupta et al. (17) compared the postoperative analgesic effect of PVB with that of SPB in fifty female patients who underwent radical mastectomy using 20 -ml bupivacaine $0.5 \%$ for both techniques. They reported that the duration of analgesia for SPB was $4 \pm 1 \mathrm{hr}$, which might be explained by low volume of local anesthetic, use of bupivacaine only and technique of the block was below serratus muscle. Moreover, Blanco et al. ${ }^{(8)}$ performed the serratus block at two different levels in the midaxillary line in four female volunteers, superficial to serratus muscle and deep underneath serratus muscle. They recorded the degree of paresthesia and they found that the mean (SD) duration of paresthesia was $12.5 \mathrm{hr}$ for the intercostal nerves and $12.96 \mathrm{hr}$ for the motor nerves after injection superficial to serratus anterior as they injected a mixture of $0.4 \mathrm{ml} . \mathrm{kg}$ levobupivacaine $0.125 \%$ mixed with 0.1 mmol.kg gadolinium

Regarding analgesia demand in our study, there were no significant differences between both groups, 1 patients only in group A needed combination of ketorolac $60 \mathrm{mg}$ in 2 divided doses and paracetamol $1000 \mathrm{mg}$ one dose after $20 \mathrm{hrs}$ from the block and no patients in group B needed rescue analgesia. In agreement with our study, Samar et al. ${ }^{(15)}$ found that time of first rescue analgesia was $20 \pm 3 \mathrm{hrs}$ and total i.v. morphine dose was $5 \pm 2 \mathrm{mg}$ in SAPB group for patients who received serratus intercostal plane block with 0.4 $\mathrm{ml} / \mathrm{kg}$ bupivacaine $0.25 \%$ plus adrenalin $5 \mu \mathrm{g} / \mathrm{ml}$ post mastectomy. Also, Jadon and Jain ${ }^{(5)}$ found that none of the patients required additional doses of analgesia with IV medication. In contrast to our study, Aly and Abd Ellatif ${ }^{(18)}$ found that time of first rescue analgesia was $6.2 \pm 1.66 \mathrm{hrs}$ and total I.V morphine dose was 18.4 $\pm 1.8 \mathrm{mg}$ in SAPB group for patients who received serratus intercostal plane block with $30 \mathrm{ml}$ bupivacaine $0.25 \%$ post thoracotomy, which might be due to that the injection was below the serratus (deep serratus block). Also, Asmaa et al. ${ }^{(19)}$ found that rescue analgesia was required by 4 patients (20\%) in the SAPB group after 16 $\mathrm{hr}$ and the total 24 hour morphine consumption by patients in the SAPB group was $10.373 \mathrm{mg}$, which might be explained by the use of levobupivacaine $(0.25 \%)$ as a sole agent and the technique of injection (below serratus).

Regarding complications in our study the use of ultrasound was introduced to improve the success rate and accuracy of the serratus anterior block and to prevent potential complications like pneumothorax, vascular puncture, nerve damage and failure or inadequate block. Moreover, there was no complications noted in group A related to the drugs used as hypotension, bradycardia, decreased R.R, nausea, vomiting or C.N.S complications. 2 cases complained of constipation in group B. In agreement with our study Jadon and Jain ${ }^{(5)}$ found that none of the patients had respiratory complications. All the patients were satisfied with the pain relief management and early discharge (within 6 days of admission). There were no reported complications from the block. Also, Nasr et al. ${ }^{(20)}$ found that there were no recorded complications in the whole studied group. In contrast to our study, Asmaa et al. ${ }^{(19)}$ found that there was 1 case of nausea in the SAPB group, which might be explained by large volume of local anesthetic ( $30 \mathrm{ml}$ of levobupivacaine followed by $5 \mathrm{ml}$ $/ \mathrm{hr}$ infusion). Moreover, Fady and Samia ${ }^{(14)}$ found that in SAPB group, there was one case suffered from nausea and vomiting, which might be also explained by large volume of local anesthetic used.

Finally regarding the technique of SABP, two different schools of thought exist regarding the site of deposition of local anesthetic in the serratus block. Blanco et al. ${ }^{(8)}$ identified two potential compartments that could be used in this block: one superficial to the serratus muscle (between serratus anterior and latissmus dosi muscle) and one deep to the muscle (between the serratus anterior and external intercostal muscles). They concluded that the superficial plane is more effective based on the distribution of the injection and sensory mapping. Fajardo et al. ${ }^{(21)}$ used Fajardo's approach and deposited the local anaesthetic below the serratus muscle. Fajardo et al. ${ }^{(21)}$ believed that greater spread is achieved if the injection is located between the serratus anterior and external intercostal muscles because respiratory movements allow the local anesthetic to be dispersed along the space due to capillary action. They 
also argued against the Blanco's approach by stating that, long thoracic nerve (LTN) may be injured if drug is injected above serratus and as this is a pure motor nerve to serratus anterior muscle (SAM) may cause winged scapula syndrome and may produce temporary palsy of the LTN. In agreement with Blanco et al. ${ }^{(8)}$, in our study we found that ultrasound guided serratus anterior plane block in the superficial plane is an effective technique for providing analgesia with rapid onset and long duration in patients with anterior and lateral rib fractures.

Many controversies also exist regarding the anatomy of the serratus anterior plane. Although, it has been described to achieve complete paresthesia of the hemi-thorax, the posterior rami, the anterior cutaneous branches of the intercostal nerves and the supraclavicular nerves are not blocked, which is achieved by epidural and paravertebral blocks ${ }^{(22)}$.

\section{CONCLUSION}

Ultrasound guided serratus anterior plane block is an effective technique for providing analgesia in patients with anterior and lateral rib fractures with fewer risks of complications.

\section{RECOMMENDATIONS}

1. Ultrasound-guided SAPB should be generalized as much as possible for all patients with fracture ribs and to be done by experienced specialist when there are no contraindications to the block.

2. Further study on SAPB especially for introduction of catheter to prolong period by continuous infusion of local anesthetic, pharmacokinetics of the drug used, comparing the efficacy of this block with other regional anesthetic techniques and evaluate the efficacy of serratus plane block for thoracic wall blockade if fractures are occurring in posterior $1 / 3$ part.

\section{REFERENCES}

1. Pressley C, Fry W, Philp A (2012): Predicting outcome of patients with chest wall injury. Am J Surg., 204: 910-4.

2. Stefano G, Ptáček R, Kuželová H (2012): Endogenous morphine: up-to-date review . Folia Biol (Praha), 58 (2): 49-56.

3. Esmailian M, Moshiri R, Zamani M (2015): Comparison of the analgesic effect of intravenous acetaminophen and morphine sulfate in rib fracture: A randomized double-blind clinical trial. Emergency, 3 (3): 99-102.

4. May L, Hillermann C, Patil S (2016): Rib fracture management. BJA Education, 16 (1): 26-32.

5. Jadon A, Jain P (2017): Serratus Anterior Plane BlockAn Analgesic Technique for Multiple Rib Fractures: A Case Series. American J Anesth Clin Res., 3 (1): 1-4.

6. Ho A, Karmakar M, Critchley L (2011): Acute pain management of patients with multiple fractured ribs: a focus on regional techniques. Curr Opin Crit Care, 7: 3237.
7. Karmakar M, Ho A (2003): Acute Pain Management of Patients with Multiple Fractured Ribs. . The Journal of Trauma: Injury, Infection, and Critical Care, 54: 615-25.

8. Blanco R, Parras T, McDonnell J et al. (2013): Serratus plane block: a novel ultrasound-guided thoracic wall nerve block. Anaesthesia, 68: 1107-13.

9. Peter F, Paul D, Christopher A et al. (2017): Case Report of Serratus Plane Catheter for Pain Management in a Patient With Multiple Rib Fractures and an Inferior Scapular Fracture. International Anesthesia Research Society, 8: 132-5.

10. Matteo B, Emanuele B, Lucia M (2016): Continuous serratus plane block in a patient with multiple rib fractures. Journal of Clinical Anesthesia, 38: 85-86.

11. Kunhabdulla N, Agarwal A, Gaur A et al. (2014): Serratus anterior plane block for multiple rib fractures. Pain Physician, 17: 553-5.

12. Rose $P$, Ramlogan $R$, Sullivan $T$ et al. (2019): Serratus anterior plane blocks provide opioid-sparing analgesia in patients with isolated posterior rib fractures: a case series. Can J Anaesth., 66 (10): 1263-1264.

13. Okmen K, Okmen B, Serkan U (2016): Serratus anterior plane (SAP) block used for thoracotomy analgesia: a case report. Korean J Pain, 29: 189-92.

14. Fady S, Samia Y, Maha A et al. (2018): Ultrasoundguided serratus anterior plane block versus thoracic paravertebral block for perioperative analgesia in thoracotomy. Saudi Journal of Anesthesia, 217 (55): 185196.

15. Samar R, Ehab A, Ehab E et al. (2018): Ultrasoundguided serratus anterior plane block versus thoracic paravertebral block for postmastectomy analgesia. Benha Medical Journal, 35: 429-36.

16. Ashok J (2017): Serratus Anterior Plane Block for Pain Relief in Multiple Fractured Ribs (MFRs)., Injection of Local Anaesthetic above the Serratus or below the Serratus? A Case Report, . Journal of Anesthesia \& CriticalCare, 7 (2): 00254.

17. Gupta K, Srikan K, Girdhar K et al. (2017): Analgesic efficacy of ultrasound guided paravertebral block versus serratus plane block for modified radical mastectomy: a randomized controlled trial. . Indian J Anaesth., 61: 3816.

18. Aly A, Abd Ellatif S (2018): Comparison of ultrasoundguided serratus plane block and thoracic paravertebral block for postoperative analgesia after thoracotomy: a randomized controlled trial. Anesthesia \& Intensive Care, 5: 314-22.

19. Asmaa E, Nasr M, Ghada M et al. (2017): UltrasoundGuided Serratus Anterior Plane Block Versus Thoracic Epidural Analgesia for Thoracotomy Pain. Journal of Cardiothoracic and Vascular Anesthesia, 31: 152-8.

20. Nasr M, Ahmed H, Rasha B et al. (2019): Ultrasound-in adjunctive analgesic with levobupivacaine for postthoracotomy pain. A prospective randomized controlled study. Dove Press Journal, 12: 1425-1431.

21. Fajardo M, López S, Diéguez $P$ (2013): A new ultrasound guided cutaneous intercostal branches nerves block for analgesia after non-reconstructive breast surgery. Cirugia Mayor Ambulatoria, 60: 18-36.

22. Tighe S, Karmakar M (2013): Serratus plane block: do we need to learn another technique for thoracic wall blockade Anaesthesia, 68: 1103-6. 